\title{
Detection and Modelling of Contacts in Explicit Finite-Element Simulation of Soft-Tissue Biomechanics
}

\author{
S.F. Johnsen - Z.A. Taylor • L. Han - Y. Hu • \\ M.J. Clarkson · D.J. Hawkes · S. Ourselin
}

the date of receipt and acceptance should be inserted later

\begin{abstract}
Purpose:

Realistic modelling of soft-tissue biomechanics and mechanical interactions between tissues is an important part of biomechanically-informed surgical image-guidance and surgical simulation. This submission details a contact modelling pipeline suitable for implementation in explicit matrix-free FEM solvers. While these FEM algorithms have been shown to be very suitable for simulation of soft tissue biomechanics and successfully used in a number of image-guidance systems, contact modelling specifically for these solvers is rarely addressed, partly because the typically large number of time steps required with this class of FEM solvers has led to a perception of them being a poor choice for simulations requiring complex contact modelling.

\section{Methods:}

The presented algorithm is capable of handling most scenarios typically encountered in image-guidance. The contact forces are computed with an evolution of the Lagrange-multiplier method first used by Taylor and Flanagan in PRONTO 3D with spatio-temporal smoothing heuristics for improved stability and edge-edge collision handling. For contact search, a bounding-volume hierarchy $(\mathrm{BVH})$ is employed that is capable of identifying self collisions by means of the surface-normal bounding cone of Volino and Magnenat-Thalmann, in turn computed with a novel formula. The BVH is further optimised for the small time steps by reducing the number of bounding-volume refittings between iterations through identification of regions with mostly rigid motion and negligible deformation. Further optimisation is achieved by integrating the self-collision criterion in the BVH creation and updating algorithms.
\end{abstract}

\section{Results:}

S.F. Johnsen · Y. Hu · M.J. Clarkson · D.J. Hawkes $\cdot$ S. Ourselin

University College London, Centre for Medical Image Computing, London, UK

Z.A. Taylor

The University of Sheffield, CISTIB Centre for Computational Imaging and Simulation Technologies in Biomedicine, Insigneo Institute for in silico Medicine, Dept. of Mechanical Engineering, Sheffield, UK

L. Han

Tongji University, School of Medicine, Shanghai, P.R. China 
The effectiveness of the algorithm is demonstrated on a number of artificial test cases and meshes derived from medical image data. It is shown that the proposed algorithm reduces the cost of BVH refitting to the point where it becomes a neglible part of the overall computation time of the simulation. It is also shown that the proposed surface-normal cone computation formula leads to about $40 \%$ fewer BVH subtrees that must be checked for self collisions compared to the widely used method of Provot. In terms of the contact-force formulation, we show that our smoothing heuristics yield more stable simulations in the presence of geometric singularities. Finally, we show with realistic image-guidance examples that the entire contact modelling pipeline can be executed within a timeframe that is the same order of magnitude as that required for standard FEM computations.

\section{Introduction}

FEM modelling has for some time now played an important role in surgical simulation [1, 29], and is finding its way into surgical guidance [3,4]. Explicit FEM solvers, particularly such based on the Total Lagrangian Explicit Dynamics (TLED), have been shown to provide a versatile and realistic means of simulating soft-tissue solid dynamics which is at the core of such guidance systems $[23,29,16]$. Their decoupling of the degrees of freedom also makes them ideal candidates for parallelisation which in recent years with the advent of generalpurpose GPUs and multi-core mainstream CPUs has proved to be a great source of costefficient execution speed [32,31].

They do, however, suffer from the inherent shortcoming of only allowing for small time steps which can make simulations involving large-deformation contact modelling prohibitively expensive mainly due to the costs associated with contact search. Another drawback is that, compared to implicit methods, little literature is available on contact modelling for these solvers. The commonly encountered ones are the penalty-force and the Lagrangemultiplier method of Taylor and Flanagan, and Heinstein et al. [12], among the force-based methods [34], and kinematic contacts that rely on a direct correction of displacements and are very efficient, but are only capable of modelling contacts between deformable and rigid bodies [9]. All of these are typically implemented as node-segment contact algorithms only capable of detecting penetration of mesh nodes into surfaces, which requires two detection passes to achieve some degree of separation of the two surfaces in contact, and even with those two passes mesh edges are still free to intersect. Node-segment methods must rely on denser meshes to avoid the latter type of mesh intersection which in turn entails more and computationally costlier time steps.

The algorithm presented in this paper was implemented as the general purpose contact modelling component of the open-source ${ }^{1}$ TLED-based FEM solver package NiftySim [16]. It attempts to carry over some of the developments made in the context of implicit contact modelling algorithms to explicit methods, such as being able to process contacts in a single pass as with segment-segment methods [28], provided the meshes in contact have a similar resolution. Spatial smoothing, which attempts to alleviate the stability issues caused by sudden changes in the direction of contact forces arising from the use of coarse, piece-wise linear contact surfaces has found widespread adoption in implicit methods [35,27], is also employed. Further stability improving modifications are introduced by gradually slowing down approaching contact surfaces in close proximity, thus adding temporal smoothing to the method.

\footnotetext{
1 http://niftysim. sourceforge.net
} 
Another major area of focus in this work is the reduction of contact-search costs through bounding volume hierarchies $(\mathrm{BVH})$ with novel, time-saving update and self-collision detection heuristics. For self-collision detection, we employ the surface-normal bounding-cone heuristics developed by Volino and Magnenat-Thalmann [33]. New formulas for the computation of the bounding cones, via Provot's recursive algorithm [26], are introduced. Another novel aspect is how the self-collision criterion is deeply integrated in determining the topology of the BVH and the decision on when to update BVH subtrees. The BVH updating algorithm is specialised for the typically small time-steps of explicit methods, in that it comprises a method for the characterisation of the deformation the simulation geometry has undergone and identification of areas of negligible deformation and rigid motion, and updating of the BVH of the latter parts by means of rigid geometrical transforms.

The proposed method is further notable due to its versatility; it allows for modelling of contacts between the surfaces of two solid meshes, self-collisions, contacts between solid and membrane meshes, and deformable bodies interacting with moving or fixed rigid ones, and a simple friction model is available, too.

This paper is organised as follows: After a brief overview of related previous work (Sect. 2), Sect. 3.1 contains an introduction of the underlying FEM algorithm, the Total Lagrangian Explicit Dynamics. This is followed by a detailed discussion of the contact modelling pipeline that can be subdivided into a relatively short part describing the contact surface data structures (Sect. 3.3) and two larger sub-sections, in the first of which the proposed collision detection method is introduced (Sect. 3.4). Novel modifications to the self-collision detection method, and the new BVH creation and update strategies are discussed in this order, in this part of the paper. The contact model is developed in Sect. 3.5; it starts with a discussion of penetration response forces for node-facet and edge-edge collisions, followed by a discussion of the rate of approach (gap-rate) proportional contact forces and the friction model. In Sect. 4, the algorithms are validated in the order in which they are presented by comparison to some alternatives that swap out some of the novel aspects for simpler or more established methods, on mostly synthetic test cases. Finally, a demonstration of the entire pipelines performance on two image-guidance problems is provided.

\section{Related Work}

Classically, the algorithms for FEM contact modelling are node-segment approaches [34], where one of the two surfaces in contact is assigned the role of the slave surface, the other is called the master surface. The only type of mesh inter-penetration node-segment approaches can resolve are those of the master surface by slave nodes, which in turn necessitates two contact search and resolution steps with alternating master-slave roles, in every time step. The underlying principle of mesh intersection handling with node-segment methods is to project slave nodes onto the nearest facet of the master surface, and check the sign of the difference between the slave node position and its projection with respect to the master surface normal. A contact response in direction of the master surface normal is then applied. Since most FEM elements are only $\mathrm{C} 0$ continuous, this approach can also lead to sudden jumps in the direction of the response experienced by a node sliding over the master surface, in turn leading to instability of the algorithm. Smoothing node-segment methods, such as the one by Wriggers and Krstulović-Opara [35] based on cubic Bézier polynomials, were introduced to remedy this issue.

Segment-segment contact algorithms were devised to overcome the need for two passes with the node-segment approach [28]. Mortar elements, originally developed for coupling 
non-conforming meshes, were introduced to the field of contact modelling to also overcome the various mathematical limitations of the early node-segment methods, mainly stability problems with implicit methods arising from non-satisfaction of the Babuska-Brezzi condition. In these methods the contacting meshes are pushed apart by a contact pressure that is interpolated over the mortar mesh. The work of Puso and Laurensen [27] introduced a mortar method for 3D and large deformations.

An interesting method suitable for any FEM or similar algorithm that assembles stiffness matrices was developed by Duriez et al. [5]. Their contact model based on Signorini's law was primarily designed with haptics in mind, and computes contact forces from the constitutive model of the bodies in contact.

An alternative to both node-segment and segment-segment methods, based on intersection volumes was devised by Heidelberger et al. [11], and significantly extended by Allard et al. [2]. By employing layered depth images (LDI) for intersection volume computation, they effectively solved collision detection and response calculation using the same method. However, the method is limited in its application to volumetric meshes.

A notable development in the area of matrix-free explicit FEM algorithms came with the Lagrange multiplier-based method employed in PRONTO 3D by Taylor and Flanagan [30], and later extended by Heinstein et al. [12].

The range of contact search algorithms proposed for FEM contact modelling is as wide as that of methods for their solution. We propose a bounding volume hierarchy (BVH) based method. These methods are very versatile and used in a wide range of applications such as cloth modelling [22], robot motion planning [8], ray tracing [17], and FEM contact modelling $[25,36]$. What makes them interesting for the application with the relatively small time steps required with explicit FEM solvers is the ability to take a more localised, selective approach to collision detection and exploitation of temporal coherence. A further advantage of employing a BVH is that it can also be used for other problems arising in surgical image guidance, such as fast point location for point-set registration purposes.

Early developments in the field of BVHs were limited to rigid or even static problems, but since the 1990s there has been a growing interest in collision detection for simulation of deformable bodies [21]. A key development came in 1994 with Volino and Magnenat-Thalmann's method [33] for efficient self-collision detection. They established two conditions under which a piece of simulated cloth could self-intersect; either the surface is folded onto itself, i.e. it has surface normals pointing in opposite directions, or there are intersecting boundary edges. Larsson and Akenine-Möller [18] devised a hybrid bottom-up/top-down $\mathrm{BVH}$ strategy for detecting collisions between deformable bodies, that reduces the number of bounding volume (BV) updates by only updating those parts of the bodies' BVHs that overlap, down to leaf level. The method, however, was not adapted for self-collision detection. They later [19] described a method for dynamically creating BVHs for triangle soups particularly suitable for such resulting from fracturing of objects. They also developed a variant of their algorithm incorporating a sweep and prune sort of all simulation primitives suitable for detecting self-collisions.

\section{Methods}

\subsection{Total Lagrangian Explicit Dynamics}

The TLED class of FEM solvers are matrix-free solvers relying on explicit central-difference time integration. They have enjoyed some success in the simulation of soft-tissue biome- 
chanics thanks to their ability to simulate large deformations, the relative ease with which complex material models can be implemented, and not least the possibility for very elegant parallel implementations [23,32,31].

The discretised equilibrium equations of TLED, neglecting damping terms, read:

$$
\frac{M}{\Delta t^{2}} U^{(t+\Delta t)}=R^{(t)}-F\left(U^{(t)}\right)-\frac{1}{\Delta t^{2}} M\left(U^{(t-\Delta t)}-2 U^{(t)}\right)
$$

where $U^{(t+\Delta t)}, U^{(t)}, U^{(t-\Delta t)}$ denote the next, current, and previous time-step displacements, respectively, $\Delta t$ is the time step size, $R$ is the external load vector, and $M$ is the lumped (diagonal) mass matrix. The term $F(U)$ represents the internal forces of the current configuration. The evaluation of the latter term does not involve the assembly of a stiffness matrix, instead internal forces are computed directly per element and subsequently accumulated for all nodes. In this work, internal forces are modelled with the neo-Hookean material model, whose strain-energy density function is given by

$$
W=\frac{G}{2}\left(\bar{I}_{1}-3\right)+\frac{K}{2}(J-1)^{2}
$$

where $G$ and $K$ denote the shear and bulk modulus of the material, respectively, and $\bar{I}_{1}=$ $J^{-2 / 3}\left(C_{11}+C_{22}+C_{33}\right)$ with $C$ denoting the left Cauchy-Green deformation tensor and $J$ is the determinant of the deformation gradient. Shell-element internal forces are computed with the EBST shell triangle of Flores and Oñate [6].

\subsection{Contact Algorithm Overview}

The algorithm is of a predictor-corrector type that first evolves the displacements with the standard TLED algorithm without any regard to contacts, then identifies intersecting or very close geometry and applies forces to correct the situation. The contact modelling pipeline comprises three major groups of routines and data structures: The contact surfaces which contain the geometry that is searched for collisions and some additional data required for contact-force application, the $B V H s$ employed in contact search, and finally the contactforce computation algorithms.

A pseudo-code overview of the algorithm including the TLED-related computations is given in Alg. 1.

\subsection{Contact Surfaces}

Contact surfaces are data structures central to the contact modelling algorithm that consist of the geometric primitives -triangles are employed in the subsequent experiments and some of the explanations- which are tested for collisions, and provide extended geometric information required in contact search such as surface normals and projection operators.

The contact surfaces associated with fixed rigid geometry are static data structures for which all normals, projection operators required for contact search and force calculation are precomputed. Moving rigid contact surface data structures are identical to their spatially fixed counterparts apart from possessing an update routine that applies the appropriate translation and/or rotation to the precomputed normals and operators.

The most important type is the deformable contact surface obtained by extracting the surface facets from the simulation solid mesh. The contact search-related members of this 


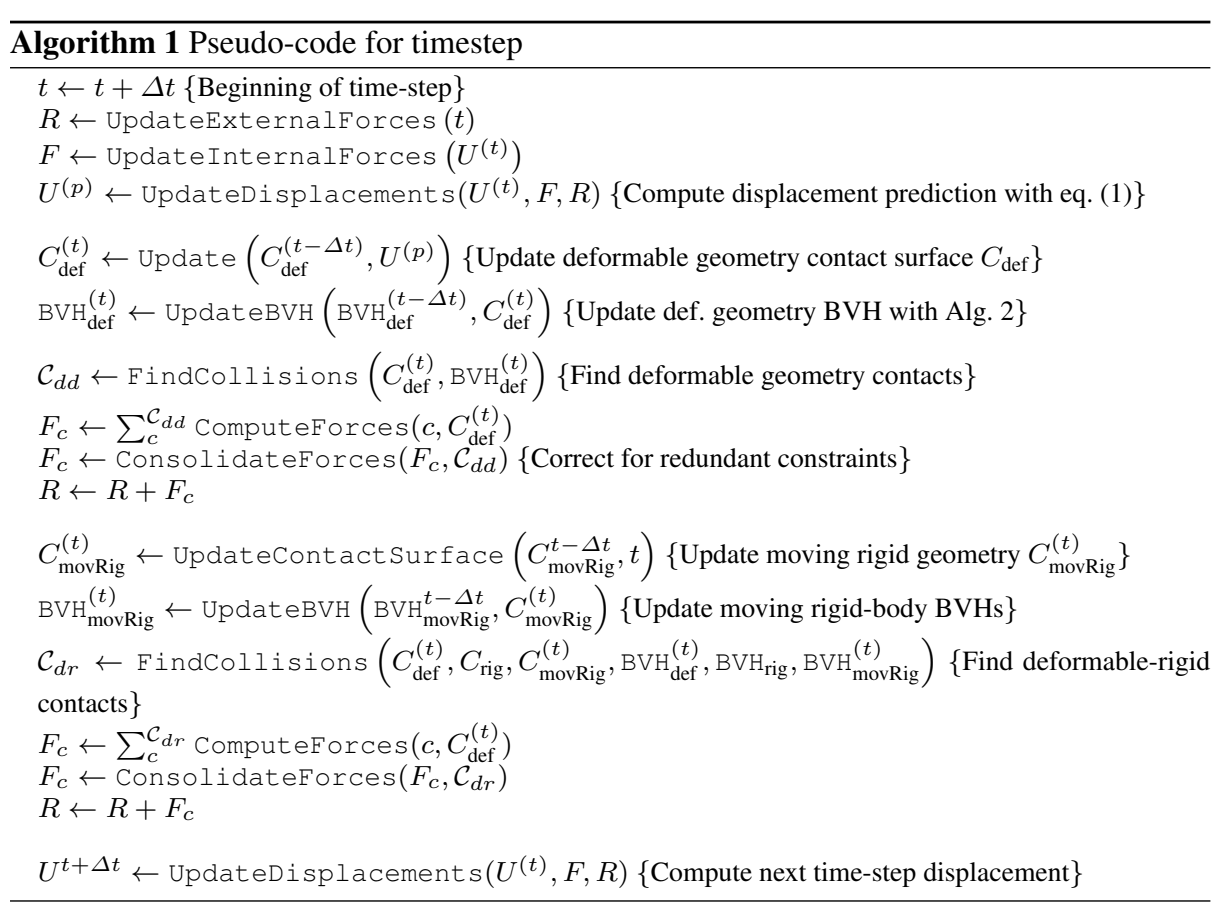

data structure are not recomputed exhaustively in every time step. Instead, a lazy evaluation with caches is employed which allows the algorithm to limit the computation of normals, etc. to regions that are in contact with or close proximity to other geometry, while not making any redundant computations. The node positions are fully updated in every time step by moving the nodes from their initial position by the predictor displacement. Contact forces which are calculated for a contact surface node are applied to the corresponding FEM node via an index lookup table that is constructed together with the surface mesh.

If the simulation contains membranes, these elements are included in the same contact surface object as the solid mesh surface facets. To account for the thickness of the membrane, two contact primitives are introduced for every membrane element, one for the top and one for the bottom. The nodes associated with these membrane contact primitives are obtained by offsetting the membrane nodes by half the thickness of the membrane in direction of the normal and its opposite, for top and bottom respectively, yielding the sandwich structure visible in Fig. 13. By having the entries in the response-force index lookup table point to the same FEM membrane node for the top and bottom node, it is ensured that contact forces are correctly incorporated in the global force vector. The updating of the node positions therefore requires a computation of the thicknesses and normals of all membrane/shell elements associated with contact primitives, in every time step. However, since these quantities are frequently either directly computed in the standard membrane internal force computation, or can be easily derived from intermediate results thereof, the costs associated with the updating of the membrane portion of the contact surface can be kept low. 


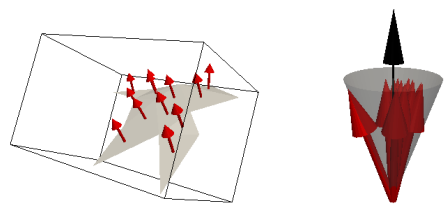

Fig. 1 Surface-normal bounding cones for self-collision detection. Left: A patch of connected geometry primitives defining a cone with the corresponding surface normals (red) and its AABB. Right: the corresponding cone, the normals it bounds (red) and the cone axis (black).

\subsection{Contact Search}

In the following, the algorithm is explained for BVHs that have a binary-tree structure. Axisaligned bounding boxes (AABB) are used for illustrations, however, the presented methods are not limited to this BV type.

At leaf level, the BVs bound one primitive each such that the primitive's vertices at the start of the time step as well as at the end of the predictor step are fully contained within it. The leaf BVs are also fitted with a safety margin $\epsilon_{B V}$ which is uniform throughout the BVH and defaults to $\frac{1}{100}\left(h_{\max }+h_{\min }\right)$ in our implementation, with $h_{\max }, h_{\min }$ being the maximum and minimum initial-configuration surface facet diameters. The purpose of this margin is to allow for some geometry deformation without the need to refit the bounding volume and to allow for the detection of primitives in close proximity.

All deformable geometry is contained in one BVH, rigid contact surfaces, moving or fixed, each have each their own BVH.

\subsubsection{Self-Collisions and Surface-Normal Bounding Cones}

The surface-normal bounding cones (NBC) are a means for identifying BVH subtrees containing geometry that is folded onto itself, i.e. has normals pointing in opposite directions, and thus potentially self-colliding [33], and are illustrated in Fig. 1. Since we mostly deal with solid elements, self-collisions resulting from intersecting mesh boundaries as described by Mezger [22] are not considered, and we treat the NBC self-collision criterion

$$
\alpha_{\mathrm{VMT}} \geq \tau_{\mathrm{VMT}}, \quad \tau_{\mathrm{VMT}} \leq \pi
$$

where $\alpha_{\mathrm{VMT}}$ is the cone opening angle and $\tau_{\mathrm{VMT}}$ the threshold above which self-collision tests are performed, as a necessary criterion for self-collision. The computation of this quantity $\alpha_{\mathrm{VMT}}$ is done recursively as part of the BVH update with a method similar to that proposed by Provot [26]. The algorithm is only applicable to connected surface primitives and computes the NBCs bottom-up, with the NBCs at leaf level being defined through the normal of the surface primitive contained in the leaf BV and an opening angle $\alpha_{\mathrm{VMT}}=0$. The employed recursive NBC formula is guaranteed to yield the narrowest parent cone for two given child NBCs. The starting point for its derivation is the realisation that an optimal parent axis $\boldsymbol{a}_{\boldsymbol{p}}$ does not only depend on the child-NBC axes, $\boldsymbol{a}_{\mathbf{1}}, \boldsymbol{a}_{\mathbf{2}}$, but also their opening angles, $\alpha_{1}, \alpha_{2}$, unlike Provot's method that only takes into account the children's axes by taking their arithmetic average. Figure 2 illustrates this idea. If $\beta$ denotes the angle between the two child axes and $\beta_{1}, \beta_{2}$ their respective angles between them and the parent axis, we want a situation where the opening angle of the parent $\mathrm{NBC} \alpha_{p}$ satisfies:

$$
\alpha_{p}=2\left(\beta_{1}+\alpha_{1} / 2\right)=2\left(\beta_{2}+\alpha_{2} / 2\right)
$$



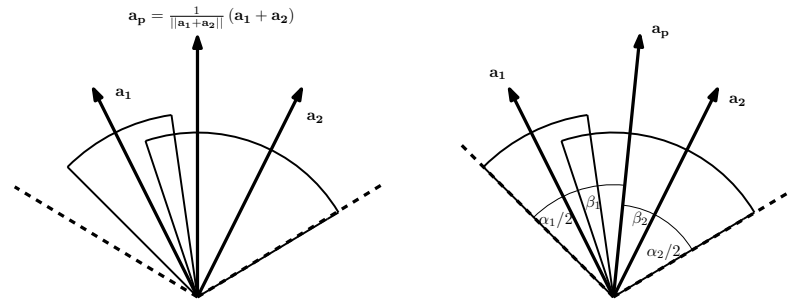

Fig. 2 Provot's NBC (left) and narrowest NBC (right). Child cones drawn with solid lines, parent NBC with dashed lines.

Using this and the definition of $\beta_{1}, \beta_{2}$ it is obtained

$$
\begin{aligned}
\beta & =\beta_{1}+\beta_{2}=\arccos \left(\boldsymbol{a}_{\mathbf{2}}{ }^{\mathrm{T}} \boldsymbol{a}_{\mathbf{1}}\right) \\
\beta_{1} & =\left(2 \beta-\left(\alpha_{1}-\alpha_{2}\right)\right) / 4, \quad \beta_{2}=\beta-\beta_{1}
\end{aligned}
$$

That in turn can be used to establish an equation system we can solve for two weights $w_{1}>0, w_{2}>0$ for computation of the parent axis from the child axes:

$$
\begin{aligned}
& w_{1}=\cos \left(\beta_{1}\right)-e \frac{\cos \left(\beta_{2}\right)-e \cos \left(\beta_{1}\right)}{1-e^{2}}, \quad \text { where } e:=\boldsymbol{a}_{\mathbf{1}}{ }^{\mathrm{T}} \boldsymbol{a}_{\mathbf{2}} \\
& w_{2}=\frac{\cos \left(\beta_{2}\right)-e \cos \left(\beta_{1}\right)}{1-e^{2}} \\
& \boldsymbol{a}_{\boldsymbol{p}}=\frac{1}{\left\|w_{1} \boldsymbol{a}_{\mathbf{1}}+w_{2} \boldsymbol{a}_{\mathbf{2}}\right\|}\left(w_{1} \boldsymbol{a}_{\mathbf{1}}+w_{2} \boldsymbol{a}_{\mathbf{2}}\right)
\end{aligned}
$$

The algorithm only performs these calculations after first checking whether one of the child cones is fully contained in the other. If so, the containing cone is adopted as the parent cone. It is therefore guaranteed that all quantities appearing in (6) lie within the valid range.

\subsubsection{BVH Generation}
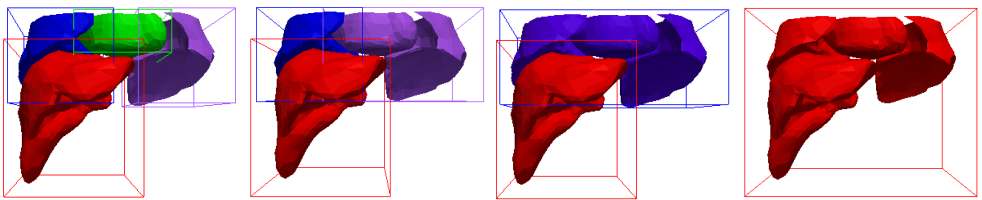

Fig. 3 Illustration of the bottom-up BV merging process. The left-most picture shows the initial state when the AABBs contain only connected geometry. The next picture shows the state after the two boxes yielding the smallest parent box have been merged. The last picture (right-most) shows the BVH root bounding all geometry. Meshes courtesy of IRCAD ${ }^{3}$.

Since the number of BVH subtrees that need to be tested for self-collisions is determined by the NBCs, the same are used to influence how the BVH is generated and updated, so as to reduce the number of $\mathrm{BV}$ intersection tests and updates. The generation process comprises two main stages, in the first of which, disconnected geometry is identified and the top part of the BVH is created from the boxes bounding these clusters, bottom-up (Fig. 3). The second stage is the top-down division of the boxes bounding the connected primitives. In order to

\footnotetext{
3 http://www.ircad.fr/softwares/3Dircadb/3Dircadb.php? Ing=en
} 
be able to apply the NBC self-collision criterion, the division process makes sure that at all stages the primitives bounded by the newly created BV remain connected. The cost function governing the assignment of primitives to child BVs, eq. (7), consists of two quantities to be minimised: the volume of the resulting $\mathrm{BV}$ and the opening angle of the NBC.

$$
\mathcal{B}_{\text {child }_{i}}^{n+1}=\mathcal{B}_{\text {child }_{i}}^{n} \cup\left\{\arg \min _{T \in \mathcal{B}_{\text {parent }}} V\left(\mathcal{B}_{\text {child }_{i}}^{n} \cup\{T\}\right)\left(1+c \cdot \alpha_{\mathrm{VMT}}\left(\mathcal{B}_{\text {child }_{i}}^{n} \cup\{T\}\right)\right)\right\}
$$

$\mathcal{B}_{\text {child }_{i}}, \mathcal{B}_{\text {parent }}$ denote the primitive sets bounded by the new children and the parent BV being split, respectively. $T \in \mathcal{B}_{\text {parent }}$ is any unassigned primitive from the parent-BV set, $V(\mathcal{B})$ is the volume of the $\mathrm{BV}$ bounding the primitive set $\mathcal{B}, \alpha_{\mathrm{VMT}}(\mathcal{B})$ the opening angle of its NBC. To be able to mix volumes and angles, we introduce the constant $c$ which is essentially arbitrary, but we determined $2 / \pi$ to be a good value. The child-primitive sets $\mathcal{B}_{\text {child }_{i}}, i \in\{1,2\}$, are initialised with the two primitives in the parent whose centroids are the farthest apart.

\subsubsection{BVH Updating}

The BVH updating algorithm only refits bounding volumes to accommodate the deformation undergone by the geometry during a time step; it does not make any changes to the BVH topology. This selective updating is achieved by means of update nodes (UN) carried over from our previous work [15]. The UNs are defined as subtree roots in which the deformation undergone by the bounded geometry is quantified to assess the need for an update of the respective BVH subtree before the next collision detection pass. They are also the starting point for any conditional top-down and the unconditional bottom-up refitting of the BVH.

An update of a subtree is required, if (1) there are potential self-collisions in the subtree, or (2) the geometry has moved so much that the bounds of the subtree's BVs are no longer valid.

In order to evaluate the first criterion, a bound on the non-rigid deformation the geometry can undergo without causing an expansion of the NBC opening angle beyond the threshold $\tau_{\mathrm{VMT}}$ is required. The bound introduced here, can be derived with a $2 \mathrm{D}$ sketch (Fig. 4): Assuming the maximum non-rigid displacement $\boldsymbol{u}_{\mathrm{NR} \text {, max }}^{\prime}$ of the bounded nodes is known, taking the smallest primitive diameter $h_{\min }$ in the set of primitives bounded by the UN, it is found that the biggest change to the primitive's normal (and thus potentially all NBCs in which it is contained) occurs if the primitives vertices both move by $\left\|\boldsymbol{u}_{\mathrm{NR}, \max }^{\prime}\right\|$ in opposite directions and perpendicularly to the plane of the primitive. The angular change to the normal $\Delta \alpha$ arising from such non-rigid deformation is

$$
\Delta \alpha=\arctan \frac{\left\|\boldsymbol{u}_{\mathrm{NR}, \max }^{\prime}\right\|}{h_{\min } / 2}
$$

The next step is to solve for $\left\|\boldsymbol{u}_{\text {NR, max }}^{\prime}\right\|$ by setting $\Delta \alpha$ to $\left(\tau_{\mathrm{VMT}}-\alpha_{\mathrm{VMT}}^{\left(t_{U}\right)}\right) / 2$, i.e., determine a safe upper bound for nodal displacement below which self-collisions can be excluded:

$$
\left\|\boldsymbol{u}_{\mathrm{NR}, \max }^{\prime}\right\|=\frac{h_{\mathrm{min}}}{2} \tan \frac{\tau_{\mathrm{VMT}}-\alpha_{\mathrm{VMT}}^{\left(t_{U}\right)}}{2}
$$

Finding the minimum primitive diameter $h_{\min }$ can be done recursively as part of the subtree update and at virtually no cost. The computation of the actual non-rigid displacements $\left\|\boldsymbol{u}^{\prime}\right\|$ requires Procrustes analysis and is significantly more costly. 


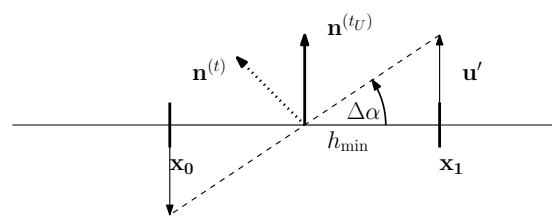

Fig. 4 In 2D: Situation leading to the largest possible change to the primitive normal for a non-rigid deformation of known magnitude $\left\|\boldsymbol{u}^{\prime}\right\|$. The nodes of the example primitive have moved in opposite directions perpendicular to the primitive's previous plane.

However, the information obtained in the Procrustes analysis, can be used to very cheaply update the subtree if update criterion 2 is satisfied but not 1 , and the BV type supports such rigid body transforms, as do e.g. oriented bounding boxes [7]. This gives rise to the update algorithm, Alg. 2. The term $\epsilon_{r}$, appearing in the pseudo-code, will be explained in section 3.5 .

The UN role is assigned to BVs at the time of BVH creation. Since subtrees potentially containing self collisions always have to be updated, it makes sense to place the update nodes well below a point in the tree where $\alpha_{\mathrm{VMT}}>\tau_{\mathrm{VMT}}$. The algorithm for the placing is a greedy one, initialising the set of UNs with the leafs of the BVH. In every iteration, it picks the two nodes whose parent has the narrowest NBC opening angle, and replaces them with their parent in the intermediate set of update nodes. This procedure continues until the minimum value of $\alpha_{\mathrm{VMT}}$ of the set of parent BVs of the current $\mathrm{UN}$ exceeds a threshold, set to $\frac{1}{2} \tau_{\mathrm{VMT}}$ in the implementation. Earlier versions of the algorithm employed a static criterion determining the UN set solely through the tree-depth of the hierarchy-nodes, in the manner Larsson and Akenine-Möller proposed for lazy updating [18]. This strategy will be used for comparison in section 4.

\subsubsection{Collision Detection}

The broad-phase collision detection is performed by recursively checking BVH (sub-) trees against each other until the bottom, i.e. the BVs bounding individual geometrical primitives, of the two BV trees is reached [22]. For self-collision detection the children of any BVH node where Eq. (3) holds true need to be checked against each other. The subsequent primitive-primitive test consists of one test for vertices against facets and one for edges against edges. The node-facet test starts by determining an initial projection and gap value, $(\tilde{\xi}, \tilde{\eta}, \tilde{g})$, of the predicted global reference-frame position of the slave vertex $\boldsymbol{x}_{\boldsymbol{s}}$ using a projection operator computed with Möller and Trumbore's method [24] with the minor modification of employing normalised facet normals instead of unnormalised one. This particular method of obtaining an initial projection is only applicable to triangular surface discretisations.

If the initial-guess projection $(\tilde{\xi}, \tilde{\eta})$ lies within the bounds of the master facet and the initial guess for the gap value, $|\tilde{g}|$, is sufficiently close to the previously nearest projection, this initial projection is improved upon with an iterative procedure that employs $\mathrm{CO}$-continuous master facet normals $\boldsymbol{n}_{\boldsymbol{m}}(\xi, \eta)$, computed from the vertex normals obtained by averaging the normals of the incident facets, and yields the final penetration depth (gap function value), $g$, and projection $\boldsymbol{x}_{\boldsymbol{m}}(\xi, \eta)$

$$
\boldsymbol{x}_{\boldsymbol{m}}(\xi, \eta):=\sum_{i \in\{\text { master facet vertices }\}} b_{i}(\xi, \eta)\left(\boldsymbol{x}_{\boldsymbol{m}}\right)_{i} \Rightarrow g:=\boldsymbol{n}_{\boldsymbol{m}}^{\mathrm{T}}(\xi, \eta)\left(\boldsymbol{x}_{\boldsymbol{s}}-\boldsymbol{x}_{\boldsymbol{m}}(\xi, \eta)\right)
$$




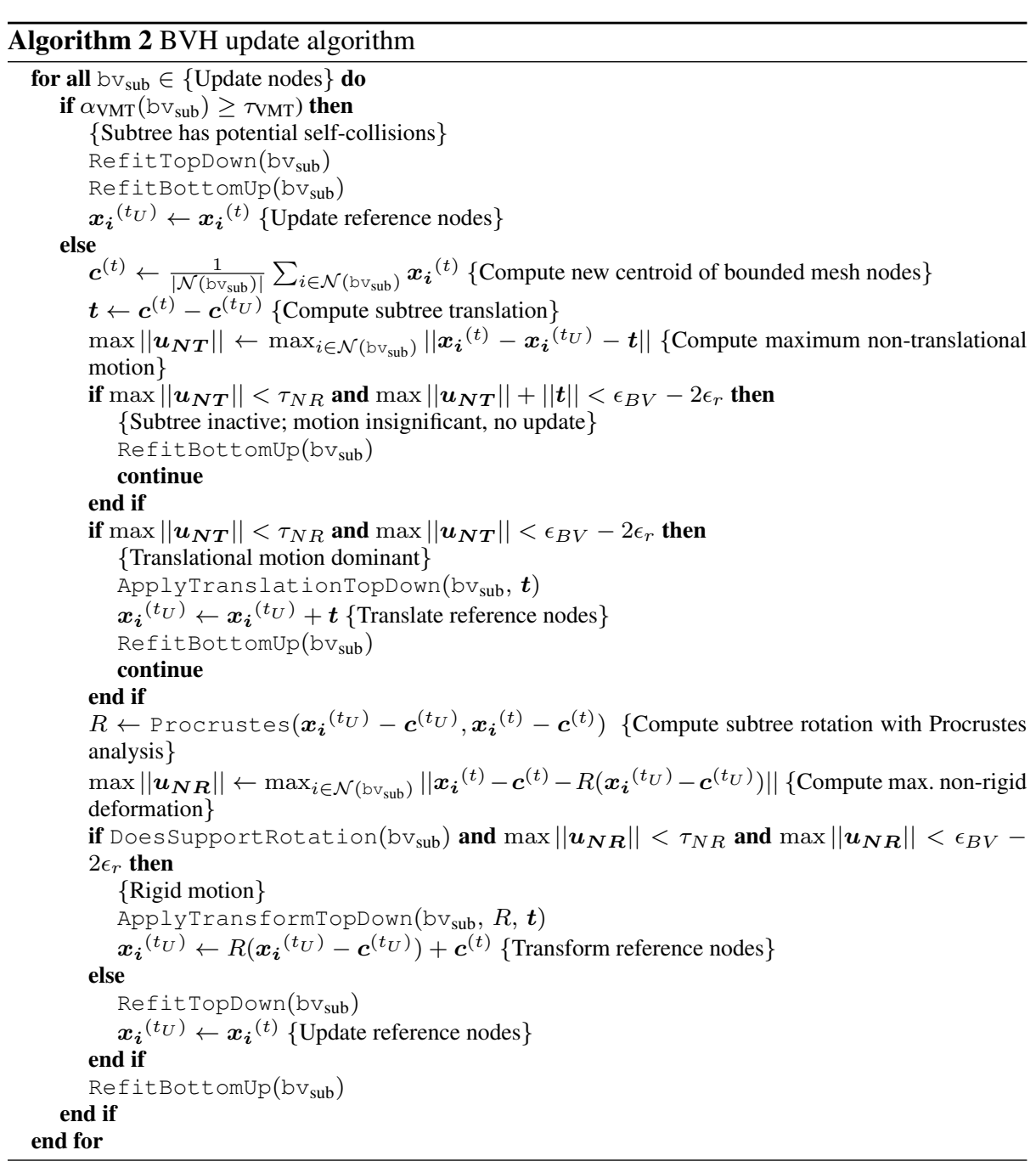

Where $\left(\boldsymbol{x}_{\boldsymbol{m}}\right)_{i}$ denotes the coordinates of the $i$-th master facet vertex, and $b_{i}(\xi, \eta)$ is a standard 2D linear shape function.

This projection $\boldsymbol{x}_{\boldsymbol{m}}(\xi, \eta)$ is the virtual master-surface node based on which the contact forces are computed (Sect. 3.5). An illustration of these quantities and their relationships is given in Fig. 5. For each slave node only the nearest projection onto a master facet is stored.

The edge-edge collision detection is performed by determining for the potentially colliding edge-edge pairs turned up by the broad-phase search their respective closest points to each other at the end of the time step. This yields the parameters $q$ and $r \in[0,1]$ that represent the positions of the closest points of the two edges, on the slave and the master edge, respectively. The difference between those closest points is subsequently projected onto master surface normal, resulting in the gap function for the edge-edge case:

$$
g=\boldsymbol{n}_{\boldsymbol{m}}^{\mathrm{T}}(r)\left(\boldsymbol{x}_{\boldsymbol{s}}{ }^{(t)}(q)-\boldsymbol{x}_{\boldsymbol{m}}{ }^{(t)}(r)\right)
$$




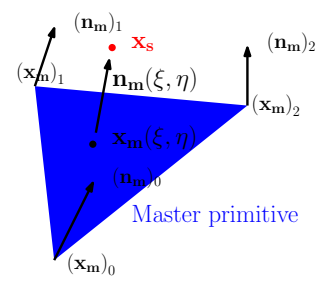

Fig. 5 Illustration of the slave-to-master projection.

\subsection{Contact-Force Calculations}

We distinguish two types of contact forces, penetration responses and gap rate-proportional forces. The former come into effect if a predictor displacement configuration leads to intersections of master and slave surfaces. The latter slow down approaching master and slave surfaces, before they can intersect.

\subsubsection{Penetration Response Calculation}

Penetrations are mathematically characterised by $g<0$, i.e. situations where the slave node lies behind the master-surface facet with respect to the master surface normal direction. The penetration response force formulas arise directly from eq. (1) with the requirement of immediate resolution of any mesh intersection and, for node-facet penetrations, are given by

$$
\begin{aligned}
\boldsymbol{f}_{\boldsymbol{s}} & =-\boldsymbol{n}(\xi, \eta) \beta_{s} \frac{m_{s} g}{\Delta t^{2}} \\
\left(\boldsymbol{f}_{\boldsymbol{m}}\right)_{i} & =\boldsymbol{n}(\xi, \eta) \beta_{m} \frac{m_{i} g \gamma_{i}(\xi, \eta)}{\Delta t^{2}}, \quad i \in \text { master facet }
\end{aligned}
$$

$\boldsymbol{f}_{\boldsymbol{s}}$ is the force applied to the penetrating slave node, $\left(\boldsymbol{f}_{m}\right)_{i}$ denotes the force applied to one of the three vertices of the penetrated master-surface primitive. $m_{s},\left(m_{m}\right)_{i}$ are the masses of the slave node and master-facet nodes, respectively.

The factor $\gamma_{i}$

$$
\gamma_{i}(\xi, \eta):=\frac{b_{i}(\xi, \eta)}{\sum_{j \in\{\text { master facet vertices }\}} b_{j}(\xi, \eta)^{2}}, \quad i \in \text { master facet }
$$

distributes the gap function value over the master facet such that at the position $\boldsymbol{x}_{\boldsymbol{m}}$ the fraction of the gap assigned to the master surface is recovered [20]:

$$
\beta_{m} g=\sum_{i \in\{\text { master facet vertices }\}} b_{i}(\xi, \eta) \gamma_{i}(\xi, \eta) \beta_{m} g
$$

The gap partitioning factor $\beta$, appearing in (12), controls how the response is split between master and slave surfaces. For contacts between rigids and deformables this would be set to 0 and 1 respectively. For inter-penetration of deformables it holds a value in $] 0,1$ [ that is computed from the masses of the nodes involved in the contact:

$$
\beta_{s}=\frac{m_{m}}{m_{s}+m_{m}}, \quad \beta_{m}=1-\beta_{s}=\frac{m_{s}}{m_{s}+m_{m}}
$$

For the purpose of gap partitioning, the mass of the virtual master node $m_{m}$ is computed with linear interpolation from the corresponding facet-vertex masses. 
With these definitions, it holds at the point of contact on the master surface:

$$
\begin{aligned}
\boldsymbol{f}_{\boldsymbol{m}} & =\sum_{i \in\{\text { master facet vertices }\}} b_{i}(\xi, \eta)\left(\boldsymbol{f}_{\boldsymbol{m}}\right)_{i} \\
& =-\boldsymbol{f}_{\boldsymbol{s}}=-\lambda \boldsymbol{n}
\end{aligned}
$$

where $\lambda$ is the Lagrange-multiplier for the constraint.

Edge-edge collision responses employ the same vertex-normals and rationale that underlie eq. (12), except that there are now two slave nodes and only two master nodes, and the $2 \mathrm{D}$ shape functions of (12) are now 1D ones.

$$
\begin{aligned}
\left(\boldsymbol{f}_{\boldsymbol{s}}\right)_{i} & =-\boldsymbol{n}_{\boldsymbol{m}}(r) \beta_{s} \frac{m_{i} \gamma_{i}(q) g}{\Delta t^{2}}, i \in \text { slave edge } \\
\left(\boldsymbol{f}_{\boldsymbol{m}}\right)_{i} & =\boldsymbol{n}_{\boldsymbol{m}}(r) \beta_{m} \frac{m_{i} \gamma_{i}(r) g}{\Delta t^{2}}, \quad i \in \text { master edge }
\end{aligned}
$$

\subsubsection{Gap Rate-Proportional Forces}

The gap-rate proportional forces are employed to achieve a degree of temporal smoothing in the contact forces. They come into effect when $0 \leq g<\epsilon_{r}$ and their relative velocity in master normal direction, their gap rate, is negative,

$$
\begin{aligned}
& \boldsymbol{n}_{\boldsymbol{m}}^{\mathrm{T}}\left(\boldsymbol{v}_{\boldsymbol{s}}-\boldsymbol{v}_{\boldsymbol{m}}(\xi, \eta)\right)<0 \\
& \boldsymbol{v}_{\boldsymbol{s}}:=\left(\boldsymbol{x}_{\boldsymbol{s}}{ }^{(t)}-\boldsymbol{x}_{\boldsymbol{s}}{ }^{(t-\Delta t)}\right) / \Delta t, \quad \boldsymbol{v}_{\boldsymbol{m}}(\xi, \eta):=\left(\boldsymbol{x}_{\boldsymbol{m}}{ }^{(t)}(\xi, \eta)-\boldsymbol{x}_{\boldsymbol{m}}{ }^{(t-\Delta t)}(\xi, \eta)\right) / \Delta t
\end{aligned}
$$

The constant $\epsilon_{r}=\frac{5}{100 \cdot 2} \epsilon_{B V}$ is chosen such that any node at distance $\epsilon_{r}$ from a master facet still lies within the safety margin of the BV and so close that any effects of the force applications are not visible in the final configuration.

The force required for velocity matching of the slave node and the virtual master node are derived from the forward-Euler increment of the velocity and momentum conservation as follows:

$$
\begin{aligned}
\boldsymbol{n}_{\boldsymbol{m}}^{\mathrm{T}}\left[\left(\boldsymbol{v}_{\boldsymbol{s}}-\boldsymbol{v}_{\boldsymbol{m}}\right)+\left(\boldsymbol{\Delta} \boldsymbol{v}_{\boldsymbol{s}}-\boldsymbol{\Delta} \boldsymbol{v}_{\boldsymbol{m}}\right)\right] & \stackrel{!}{=} 0 \\
\boldsymbol{\Delta} \boldsymbol{v}_{\boldsymbol{s}}=\frac{\Delta t}{m_{s}} \boldsymbol{n}_{\boldsymbol{m}} \dot{\lambda}, \quad \boldsymbol{\Delta} \boldsymbol{v}_{\boldsymbol{m}} & =-\sum_{i \in \text { master facet }} b_{i} \frac{\Delta t}{m_{i}} \gamma_{i} \boldsymbol{n}_{\boldsymbol{m}} \dot{\lambda}
\end{aligned}
$$

This gives rise to the following formula for the force's magnitude $\dot{\lambda}$

$$
\dot{\lambda}=-\frac{\boldsymbol{n}_{\boldsymbol{m}}^{\mathrm{T}}\left(\boldsymbol{v}_{\boldsymbol{s}}-\boldsymbol{v}_{\boldsymbol{m}}\right)}{\Delta t\left(1 / m_{s}+\sum_{i} b_{i} \gamma_{i} / m_{i}\right)}
$$

The applied force gradually increases as the distance between the surfaces decreases, and full velocity-matching is performed when there is zero distance between the slave node and its projection onto the master surface

$$
\boldsymbol{f}_{\boldsymbol{s}}=-\left(1-g / \epsilon_{r}\right) \boldsymbol{n}(\xi, \eta) \dot{\lambda}, \quad\left(\boldsymbol{f}_{m}\right)_{i}=\left(1-g / \epsilon_{r}\right) \boldsymbol{n}(\xi, \eta) \dot{\lambda} \gamma_{i}
$$

The corresponding formula for $\dot{\lambda}$, for edge-edge contacts reads:

$$
\dot{\lambda}=-\frac{\boldsymbol{n}_{\boldsymbol{m}}^{\mathrm{T}}\left(\boldsymbol{v}_{\boldsymbol{s}}-\boldsymbol{v}_{\boldsymbol{m}}\right)}{\Delta t\left(\sum_{i \in \text { master edge }} b_{i} \gamma_{i} / m_{i}+\sum_{i \in \text { slave edge }} b_{i} \gamma_{i} / m_{i}\right)}
$$




\subsubsection{Friction}

Equation (20) can be used in Coulomb's model to simulate friction by substituting the relative tangential velocity for the gap rate. Modelling friction with the proposed contact modelling method only requires keeping track of the active constraints in a given time step and the associated normal forces, and application of the forces computed from

$$
\boldsymbol{f}=\left\{\begin{array}{l}
-\lambda_{T} \boldsymbol{\Delta} \boldsymbol{v}_{\boldsymbol{T}} /\left\|\boldsymbol{\Delta} \boldsymbol{v}_{\boldsymbol{T}}\right\|, \text { if } \lambda_{T}<\mu\left\|\boldsymbol{f}_{\boldsymbol{N}}\right\| \\
\mu\left\|\boldsymbol{f}_{\boldsymbol{N}}\right\| \boldsymbol{\Delta} \boldsymbol{v}_{\boldsymbol{T}} /\left\|\boldsymbol{\Delta} \boldsymbol{v}_{\boldsymbol{T}}\right\|, \text { otherwise }
\end{array}\right.
$$

with $\mu$ being the friction coefficient, $f_{N}$ the normal forces applied to the respective node, and

$$
\begin{aligned}
\boldsymbol{\Delta} \boldsymbol{v}_{\boldsymbol{T}} & :=\left(\boldsymbol{v}_{\boldsymbol{s}}-\boldsymbol{v}_{\boldsymbol{m}}\right)-\boldsymbol{n}_{\boldsymbol{m}}^{\mathrm{T}}\left(\boldsymbol{v}_{\boldsymbol{s}}-\boldsymbol{v}_{\boldsymbol{m}}\right) \cdot \boldsymbol{n}_{\boldsymbol{m}} \\
\lambda_{T} & :=\frac{\left\|\boldsymbol{\Delta}_{\boldsymbol{T}}\right\|}{\Delta t\left(1 / m_{s}+b_{i} \gamma_{i} / m_{i}\right)}
\end{aligned}
$$

\subsubsection{Contact-Force Consolidation}

Since nodes can be involved in multiple contacts, e.g. multiple edge-edge contacts or masterfacet vertices being part of multiple node-facet contacts, the contact forces must be consolidated. This can be relatively easily accomplished if the indices subject contact constraints are being kept track of as the responses are computed. The option chosen in our algorithm is that of computing for every node with an active contact constraint the mean direction of the contact forces and applying the maximum projection over all response forces in that direction. Alg. 3 contains a pseudo-code description of the consolidation algorithm.

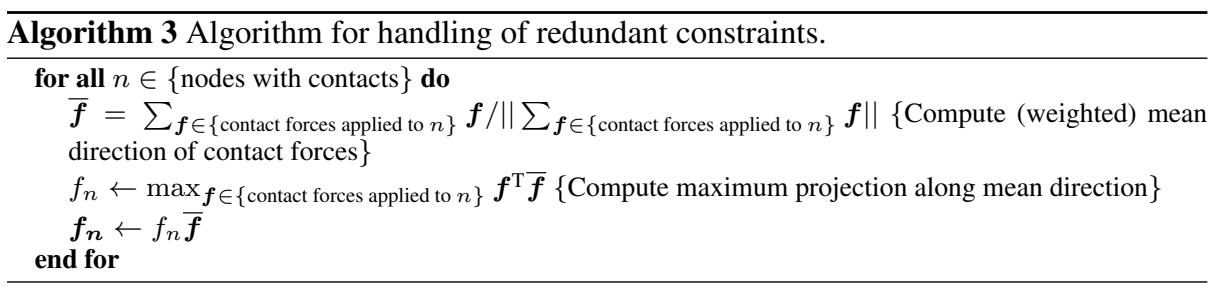

With the right data structures, this algorithm can be implemented with an $O\left(N_{\text {node-facet }}+\right.$ $\left.N_{\text {edge-edge }}\right)$ runtime complexity, where $N_{\text {node-facet }}$ and $N_{\text {edge-edge }}$ are the number of node-facet and edge-edge contacts, respectively.

\section{Experiments}

Our objective in this section is to show the inherent advantages of the individual heuristics introduced in this paper over a number of alternatives. The subsequent evaluations are based on a single-threaded $\mathrm{C}++$ implementation of the algorithm described in the previous section. The FEM calculations were done with NiftySim's CPU solver [16]. The timings were obtained by surrounding individual parts of the code representing the major stages of the contact modelling pipeline with calls to the $\mathrm{C} \mathrm{clock}$ function and accumulation. The timings were obtained on a workstation equipped with an Intel Core i7 $2600 \mathrm{~K}$ processor and $8 \mathrm{~GB}$ of RAM. 


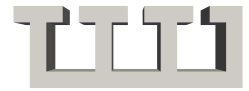

Zipper: "Zipper" geometry twisted and compressed through force boundary conditions $\left(F_{x}= \pm 30, F_{y}= \pm 5, F_{z}=-10\right)$. Initial configuration shown in left, final configuration shown in right picture.

Dimensions: $35 \times 7 \times 12.5$.

Mesh: 712 surf. facets, 358 surf. nodes, 1587 solid el's, 485 solid nodes.

Material: $G=10, K=40, \rho=100$ Total time: $T=100$.

Time step size: $\Delta t=0.075$
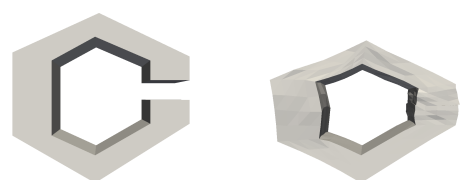

C: C shape being closed by application of displacement boundary conditions to upper part of geometry $\left(u_{z}=-3.5\right)$. Dimensions: $22.5 \times 10 \times 21.5$.

Mesh: 960 surf. facets, 482 surf. nodes, 2320 solid el's, 667 solid nodes.

Material: $G=10, K=40, \rho=1$. Total time: $T=2$.

Time step size: $\Delta t=0.001$.

Fig. 6 Simulation geometry and settings used in validation of the proposed cone formula.

\subsection{Self-Collision Detection Cones}

The first two experiments are aimed at quantifying the effects of the formula for computation of the NBCs, described in Sect. 3.4.1. To this end, it is compared to the recursive method of Provot [26]. The geometry and simulation settings of the simulations are given in Fig. 6. Due to the mostly artificial nature of the geometry, units have been omitted, but all parameter values can be assumed to be specified in compatible units, e.g. m, s, Pa, etc. The geometry was generated with AutoCAD ${ }^{4}$ and solid meshing was done with $\mathrm{GMSH}^{5}$. Most of the experiments are once run with a binary $\mathrm{AABB}$ hierachy $(\mathrm{AABB} 2)$ and once with a 4-nary BVH (AABB4).

The results in terms of the average number of BVH subtrees that need checking for selfcollisions per time step, BVs that needed refitting, and the total time spent updating BVHs and searching for contacts, for these two simulations are given in Tab. 1.

A reduction in the $40 \%$ ballpark in the number of BVH subtrees that need to be visited for self-collision detection (first column in Tab. 1) is achieved with our proposed formula in these two test cases. Further, since our BVH refitting and construction algorithms take into account the NBC opening angle, the number of BVs that are refitted is about $10 \%$ lower with our NBC computation method (second column in Tab. 1). The latter effect can mostly make up for the slight increase in computational costs that comes with our formulation. These findings are consistent across the two considered BVH orders.

\subsection{BVH Refitting Strategy}

The second set of experiments deals with the evaluation of the proposed BVH updating strategy. Most of the geometry used in these experiments is again artificial and created with Meshlab $^{6}$, except the test case containing a liver and diaphragm whose geometry was ex-

\footnotetext{
4 http://usa.autodesk.com/autocad/

5 http://www.geuz.org/gmsh/

6 http://meshlab. sourceforge. net/
} 
(a) Our cone method

\begin{tabular}{|c||c|c|c|c|}
\hline Experiment & $\begin{array}{l}\text { Avg. } \\
\text { BVH s. of } \\
\text { subtrees } \\
\text { with } \\
\text { self-collisions }\end{array}$ & $\begin{array}{l}\text { Avg. N. of refit- } \\
\text { ted BVs / time } \\
\text { step (tot. N. of } \\
\text { BVs) }\end{array}$ & $\begin{array}{l}\text { Contact search } \\
\text { total time (s) }\end{array}$ & $\begin{array}{l}\text { BVH update to- } \\
\text { tal time (s) }\end{array}$ \\
\hline C AABB2 & 32.9 & $216.2(1919)$ & 4.35 & 0.129 \\
\hline C AABB4 & 17.4 & $101.4(1410)$ & 5.01 & 0.117 \\
\hline Zipper AABB2 & 50.3 & $229.4(1423)$ & 1.43 & 0.0936 \\
\hline Zipper AABB4 & 22.9 & $167.3(1065)$ & 1.49 & 0.1 \\
\hline
\end{tabular}

(b) Provot's method

\begin{tabular}{|c||l|c|c|c|}
\hline Experiment & $\begin{array}{l}\text { Avg. N. of } \\
\text { BVH subtrees } \\
\text { with potential } \\
\text { self-collisions }\end{array}$ & $\begin{array}{l}\text { Avg. N. of refit- } \\
\text { ted BVs / time } \\
\text { step (tot. N. of } \\
\text { BVs) }\end{array}$ & $\begin{array}{l}\text { Contact search } \\
\text { total time (s) }\end{array}$ & $\begin{array}{l}\text { BVH update to- } \\
\text { tal time (s) }\end{array}$ \\
\hline C AABB2 & 57.5 & $234.5(1919)$ & 5.04 & 0.113 \\
\hline C AABB4 & 28.5 & $121.3(1410)$ & 5.49 & 0.103 \\
\hline Zipper AABB2 & 87.7 & $254(1423)$ & 1.62 & 0.0823 \\
\hline Zipper AABB4 & 45.2 & $220.7(1065)$ & 1.72 & 0.0898 \\
\hline
\end{tabular}

Table 1 Results from the comparison of our cone computation method with that of Provot.

tracted from volunteer MRI data with Slicer $3 \mathrm{D}^{7}$. The "C" test case from the previous section is also used in these experiments. The new test simulations are summarised in Fig. 7.

The results for these experiments can be found in Tab. 2. No results are available for Larsson and Akenine-Möller's method on the "rigid bar" test case, since the method is not defined for rigid-deformable contacts. Similarly, while technically it can be easily extended to applications in self-collision detection, its authors did never intend for it to be used in that way, and its performance is very poor and provides little insight in this context. Therefore, there are no results for the "C" test case in Tab. 2(b), either.

The first observation that can be made from these results is that our proposed BVH updating strategy leads to a general reduction in BV refitting and, ultimately, in overall computation time, over both exhaustive refitting and Larsson and Akenine-Möller's update strategy. This effect is more pronounced on higher resolution meshes and problems not involving self-collisions. The latter is most likely due to the dominant effect of contact search on the overall compuation costs of self-collision problems. The reduction in BVH refitting costs over exhaustive refitting approaches one order of magnitude with our method, on the larger problems, despite the not insignificant computational overhead introduced by the deformation analysis.

\subsection{Scaling}

The aim here is to show how the performance of our proposed NBC computation formula and BVH update method change with increasing mesh resolution. To this end, the zipper self-collision test case is taken and the mesh refined in 5 steps. The Zipper test case was chosen for this experiment due to the relatively large deformation and because all deformation stems from force application, there is hence no bias towards translational movement of geometry which might give our proposed method an unfair advantage.

\footnotetext{
7 http://www.slicer.org/
} 


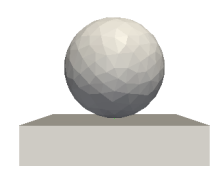

Ball-slab: Ball being pushed against a deformable slab through displacement boundary conditions (vertically by $u_{z}=-0.4$ ).

Dimensions: Ball radius $=1.2$, slab: $3.15 \times 3.15 \times 1.2$.

Mesh: 1500 surf. facets, 754 surf. nodes, 3284 solid el's, 981 solid nodes.

Material: $G=100, K=400, \rho=20$. Time: $T=2, \Delta t=0.001$
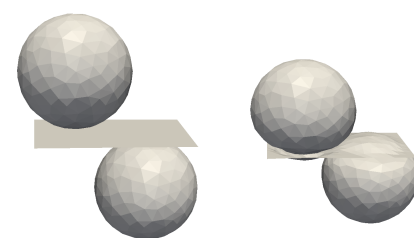

Two spheres: Two spheres pushed against a membrane via force constraints $\left(F_{z}= \pm 1\right)$

leading to two-sided contact. Only membrane mid-surface shown

Dimensions: sphere radius $=0.7$, membrane dimensions $=2 \times 1 \times 0.15$.

Mesh: $2 \times 700$ sphere surf. facets, $2 \times 252$

sphere surf. nodes, $2 \times 789$ solid el's, $2 \times 278$ solid nodes, 400 membr. el's, 231 membr. nodes.

Material: $G_{\text {sphere }}=200, K_{\text {sphere }}=600$, $G_{\text {membrane }}=1200, \rho_{\text {sphere }}=25$,

$\rho_{\text {membrane }}=200$; membrane is incompressible Time: $T=0.5, \Delta t=10^{-4}$

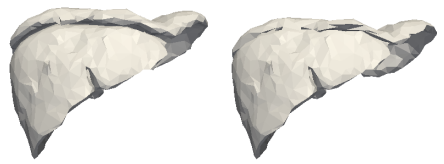

Diaphragm/liver: Diaphragm pushed against liver by displacement boundary conditions $\left(u_{z}=-0.2\right)$.

Mesh: 2400 surf. facets, 1202 surf. nodes, 4921 solid el's, 1448 solid nodes.

Material: $G=15, K=50, \rho=200$. Time: $T=1, \Delta t=0.0025$
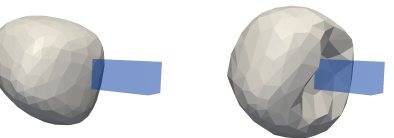

Rigid bar: An irregularly shaped body (grey) is pushed via displacement boundary conditions $\left(u_{x}=0.5\right)$ on its back against a rigid bar (semi-transparent, blue)

Dimensions: $0.91 \times 1 \times 1.34$ (deformable body AABB), $0.5 \times 0.25 \times 0.25$ (rigid body)

Mesh: 600 surf. facets, 302 surf. nodes, 1184

solid el's, 359 solid nodes, 120 rigid facets, 83 rigid nodes.

Material: $G=0.05, K=0.1, \rho=0.5$

Time: $T=2, \Delta t=0.0005$

Fig. 7 Experiments used in BVH update-strategy validation.

The first experiment looks at the NBCs. The same simulation is run once with Provot's recursive method and once with ours and the average number of BVH subtrees that need checking for self-collisions and the average time required for contact modelling operations per time step are recorded. The results for binary and 4-nary AABB hierarchies can be found in Fig. 8. The timing values include the time required for all BVH and contact surfaceupdate, as well as collision-detection and response computations.

With both types of hierarchies there is a clear divergence in the number of sub-trees that need checking for self-collisions, which in turn manifests in noticeably lower computation times for our method at higher mesh resolutions. That the divergence in the time required for contact modelling operations, in turn dominated by the contact search, does not diverge stronger can likely be explained with the subtree pairs that need checking with both types of NBCs having their roots higher up in the hierarchy and thus being more time-consuming to traverse.

The second experiment, looking at the proposed BVH update strategy, is mostly identical except what is recorded is the number of refitted BVs and the average per time-step BVH refitting costs in milliseconds. The results for exhaustive refitting are included for reference. The results for binary and 4-nary AABB hierarchies can be found in Fig. 9. 
(a) Our update strategy

\begin{tabular}{|c||c|c|c|}
\hline Experiment & $\begin{array}{l}\text { Avg. number of refit- } \\
\text { ted BVs / time step } \\
\text { (tot. N. of BVs) }\end{array}$ & $\begin{array}{l}\text { BVH update: avg. } \\
\text { time (ms) / time step }\end{array}$ & $\begin{array}{l}\text { Total computation } \\
\text { time (s) }\end{array}$ \\
\hline Ball-slab AABB2 & $111.9(2999)$ & 0.0433 & 2.91 \\
\hline Ball-slab AABB4 & $50.1(2265)$ & 0.0407 & 4.02 \\
\hline C AABB2 & $216.2(1919)$ & 0.0675 & 6.73 \\
\hline C AABB4 & $101.4(1410)$ & 0.0606 & 1.65 \\
\hline Liver-diaphragm AABB2 & $659.7(4799)$ & 0.202 & 1.67 \\
\hline Liver-diaphragm AABB4 & $412.4(3659)$ & 0.196 & 5.81 \\
\hline Two spheres AABB2 & $160.5(3599)$ & 0.0488 & 1.15 \\
\hline Rigid bar AABB2 & $75.3(1199)$ & 0.0229 & 1.14 \\
\hline Rigid bar AABB4 & $44.7(904)$ & 0.0228 & \\
\hline
\end{tabular}

(b) Larsson and Akenine-Möller's update strategy

\begin{tabular}{|c||c|c|c|}
\hline Experiment & $\begin{array}{l}\text { Avg. number of refit- } \\
\text { ted BVs / time step } \\
\text { (tot. N. of BVs) }\end{array}$ & $\begin{array}{l}\text { BVH update: avg. } \\
\text { time (ms) / time step }\end{array}$ & $\begin{array}{l}\text { Total computation } \\
\text { time (s) }\end{array}$ \\
\hline Ball-slab AABB2 & $898.9(2999)$ & 0.129 & 3.11 \\
\hline Ball-slab AABB4 & $581.9(2265)$ & 0.0973 & 4.14 \\
\hline Liver-diaphragm AABB2 & $2729.6(4799)$ & 0.430 & 1.77 \\
\hline Liver-diaphragm AABB4 & $2035.7(3659)$ & 0.348 & 6.67 \\
\hline Two spheres AABB2 & $1039.1(3599)$ & 0.143 & 6.31 \\
\hline
\end{tabular}

(c) Exhaustive refitting

\begin{tabular}{|c||c|c|c|}
\hline Experiment & $\begin{array}{l}\text { Avg. number of refit- } \\
\text { ted BVs / time step } \\
\text { (tot. N. of BVs) }\end{array}$ & $\begin{array}{l}\text { BVH update: avg. } \\
\text { time (ms) / time step }\end{array}$ & $\begin{array}{l}\text { Total computation } \\
\text { time (s) }\end{array}$ \\
\hline Ball-slab AABB2 & $2999(2999)$ & 0.301 & 3.49 \\
\hline Ball-slab AABB4 & $2265(2265)$ & 0.257 & 4.84 \\
\hline C AABB2 & $1919(1919)$ & 0.214 & 6.17 \\
\hline C AABB4 & $1410(1410)$ & 0.184 & 6.66 \\
\hline Liver-diaphragm AABB2 & $4799(4799)$ & 0.657 & 1.87 \\
\hline Liver-diaphragm AABB4 & $3659(3659)$ & 0.542 & 7.61 \\
\hline Two spheres AABB2 & $3599(3599)$ & 0.408 & 1.65 \\
\hline Rigid bar AABB2 & $1199(1199)$ & 0.146 & 1.56 \\
\hline Rigid bar AABB4 & $904(904)$ & 0.125 & \\
\hline
\end{tabular}

Table 2 Results of comparison of BVH update strategies.

The most striking result is, as the number of surface primitives increases almost tenfold, the number of updated BVs remains almost constant with our strategy. This can be explained with the UN being placed based purely on geometric criteria. Meanwhile, the costs of exhaustively refitting the BVH approaches $50 \%$ of the total contact-modelling costs observed for our algorithm in Fig. 8. From these plots it can also be seen that the savings in terms of overall computation time with AABB4 over AABB2 and exhaustive refitting, observable in Tab. 2, can be attributed only to the fewer refitted BVs with the higher order BVH.

\subsection{Contact Forces}

This series of experiments looks at different ways of modelling contacts. Since stability problems frequently arise around geometrical singularities (sharp edges, corners), the be- 

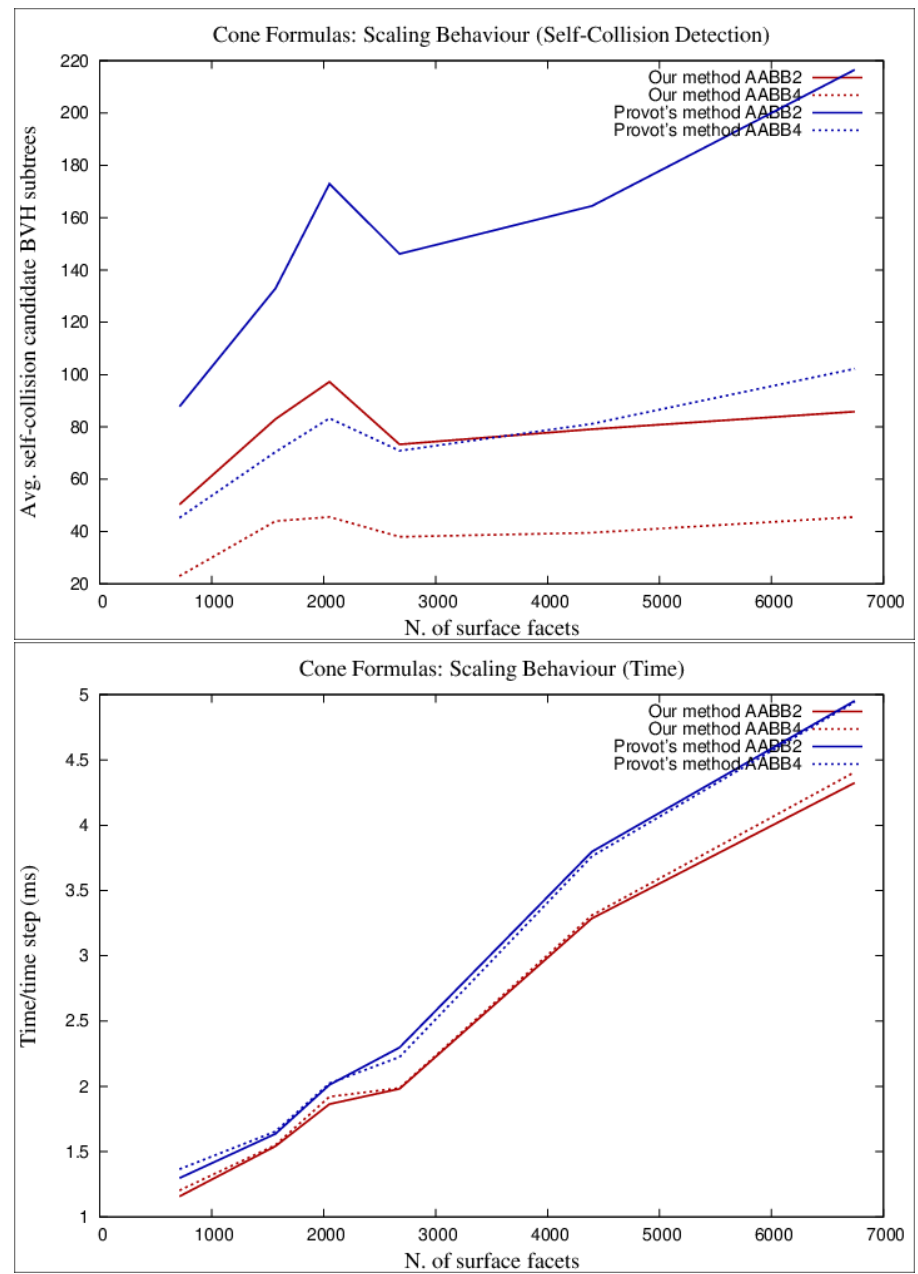

Fig. 8 Comparison of our method of NBC computation to that of Provot. Top: number of self-collision candidate subtrees plotted against mesh resolution. Bottom: Corresponding contact modelling computation time per time step.

haviour of our methods for improving stability of the contact are investigated in such a context. To this end its performance is compared to a) an otherwise identical method that computes the response forces from constant facet-normals, and b) an otherwise identical method without gap-rate forces.

In the first experiment, whose setup is depicted in Fig. 10, a moving part with a sawtoothed profile is dragged over a smooth surface. The top of the moving part is subjected to displacement constraints that are linearly ramped up from 0 to -60 over the course of the simulation, resulting in a constant velocity of the part. The constitutive model of both parts is neo-Hookean; the moving part is significantly stiffer, with $G=20$ and $K=80$, than the fixed part, with $G=1$ and $K=4$. The mass density for both meshes is $\rho=1$. The meshes consist 4311 tetrahedral elements and 870 nodes, in the case of the one with the saw-toothed 

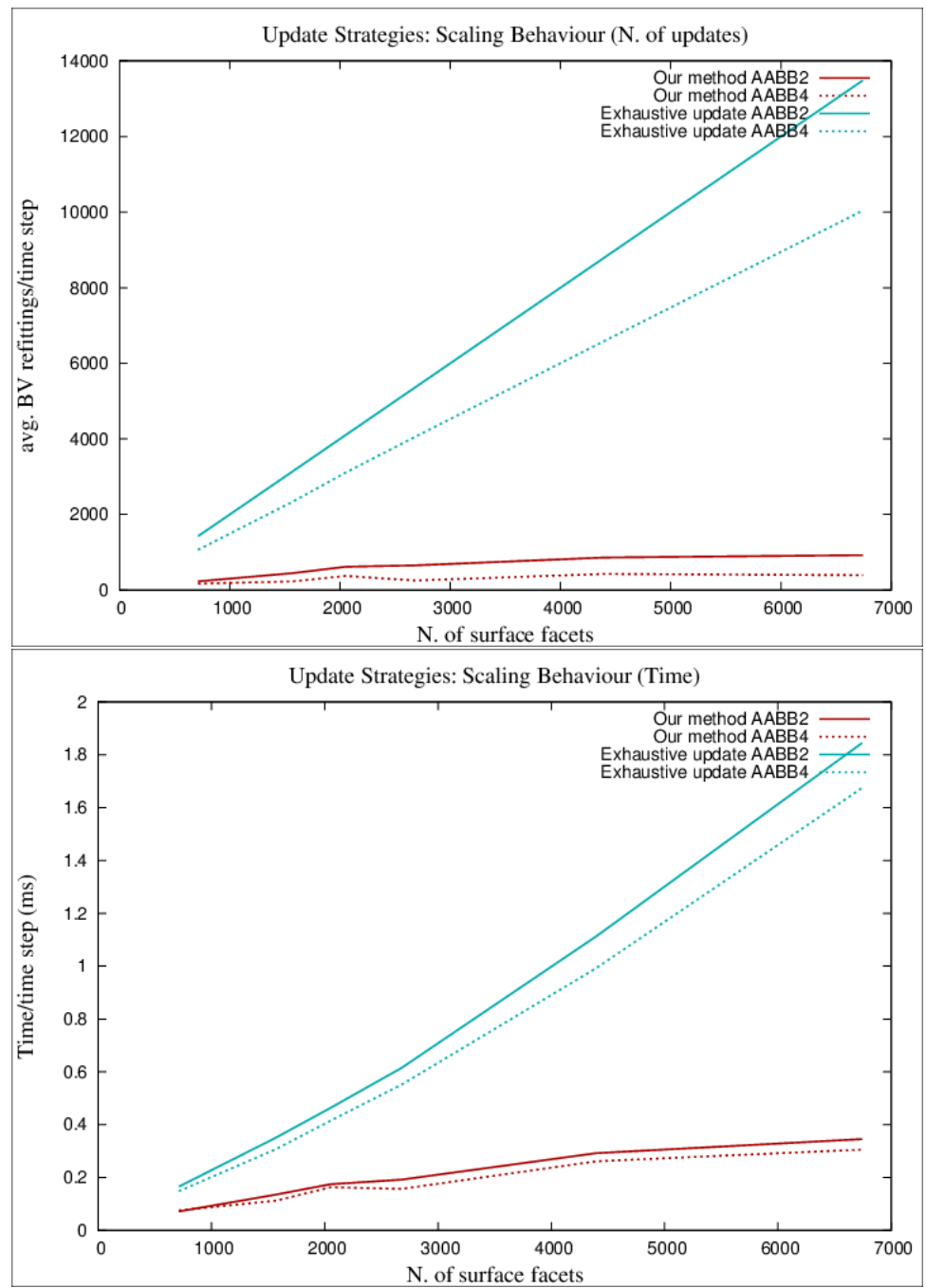

Fig. 9 Update strategy scaling behaviour: our method, and exhaustive refitting. Top: average number of refitted BVs / time step. Bottom: average BVH update time / time step.

profile, and the fixed part is made up of 4178 tetrahedral elements and 890 nodes. The time step size is held constant at $\Delta t=2.5 \times 10^{-4}$.

In the experiment, the total simulation time is gradually reduced, thus leading to an increase in velocity of the moving part. The experiment starts with a total simulated time of $T=1.5$ and $T$ is reduced in steps of $0.1 \mathrm{~s}$, and we observe the ability to converge of the different methods. Tab. 3 shows whether the methods converge for a given value of $T$.

In the second experiment, two square boxes of $1 \times 1 \times 1$ are being pushed past each other (Fig. 11). One box is again subject to linearly increasing displacement constraints, the other is held in place, and their placement and motion is such that there is a large area of overlap when they touch, of $0.4 \times 0.4$. Again the simulation time is gradually reduced. The box meshes are identical and consisting of 555 nodes and 2765 tetrahedral elements. The 


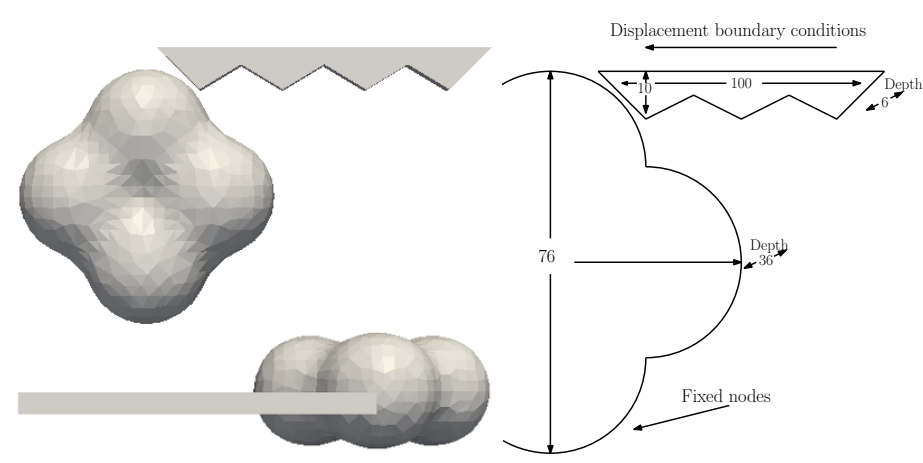

Fig. 10 Left: Initial configuration of first simulation as seen from side and above. Right: Simulation setup sketch with mesh dimensions and indication of location of boundary conditions.

\begin{tabular}{|c||c|c|c|}
\hline$T$ & Our method & No-rate responses & Constant normals \\
\hline 1.5 & Converged & Converged & Converged \\
\hline 1.4 & Converged & Converged & Converged \\
\hline 1.3 & Converged & Converged & Converged \\
\hline 1.2 & Converged & Diverged & Converged \\
\hline 1.1 & Converged & Diverged & Diverged \\
\hline 1.0 & Diverged & Diverged & Diverged \\
\hline
\end{tabular}

Table 3 Convergence of different response types with different values for $T$ in the first contact response experiment

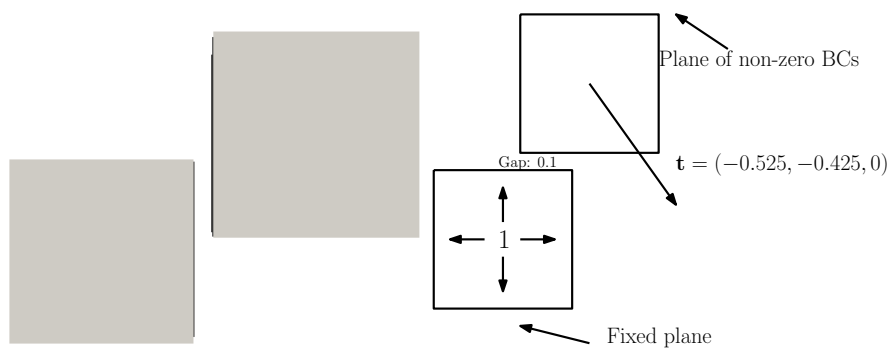

Fig. 11 Left: Initial configuration of second simulation as seen from side. Right: Simulation setup sketch with mesh dimensions and indication of location of boundary conditions.

material parameters are uniformly $G=2.5, K=10$, and $\rho=1$. In this experiment, $T$ is initially 15 and reduced in steps of 1 .

Tab. 4 shows which methods converged with a given $T$ value.

In both experiments, the last method to diverge is the one with both spatial and temporal smoothing. Aside from the lower number of time steps required in quasi-static problems, the ability of the method to converge for a given setting of $T$ is of great importance in applications where transient effects are of interest.

\subsection{Examples from Image-Guidance}

In this section two quasi-static image-guidance examples of FEM contact modelling based on actual patient data are presented with the primary aim of demonstrating the proposed al- 


\begin{tabular}{|c||c|c|c|}
\hline$T$ & Our method & No-rate responses & Constant normals \\
\hline 15 & Converged & Converged & Diverged \\
\hline 14 & Converged & Converged & Diverged \\
\hline 13 & Converged & Converged & Diverged \\
\hline 12 & Converged & Diverged & Diverged \\
\hline 11 & Diverged & Diverged & Diverged \\
\hline
\end{tabular}

Table 4 Convergence of different response types with different values for $T$ in the second contact response experiment

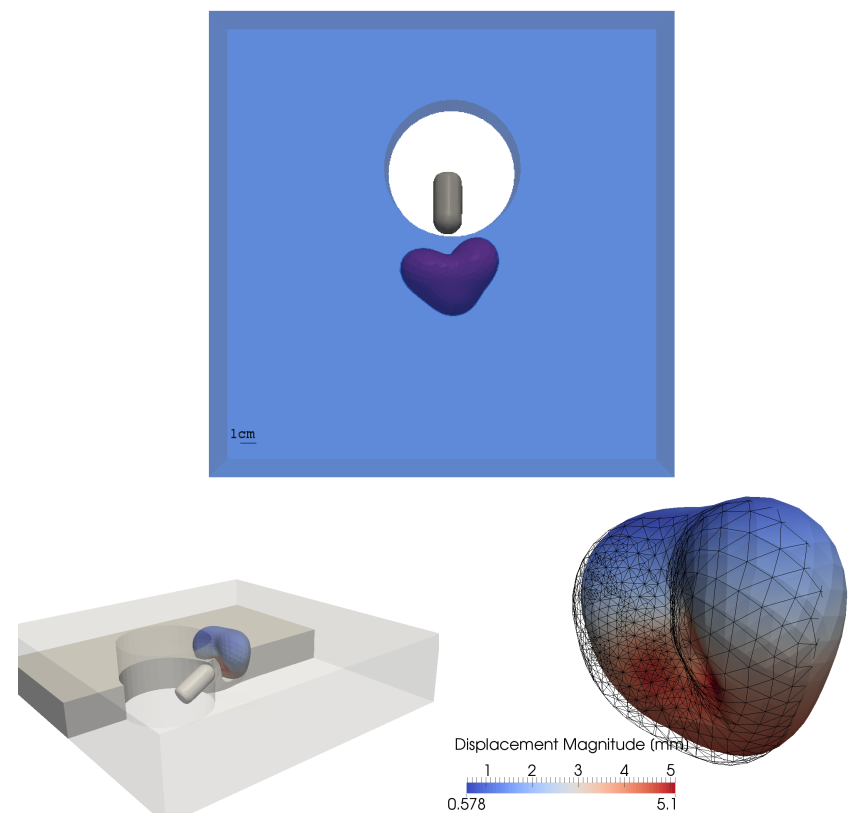

Fig. 12 Top: Initial-configuration geometry of the prostate example. Prostate shown in purple, surrounding tissue in blue, TRUS probe mesh in grey. Bottom left: Cutaway view of simulation final configuration. Bottom right: prostate final-configuration posterior $3 / 4$ view with initial configuration overlaid (wireframe).

gorithm's performance on high-resolution meshes encountered in TLED's main application area. The code is sequential and uses binary AABB hierarchies for contact search. The first one is a reconstruction of the deformation caused to the prostate by the transrectal ultrasound (TRUS) probe used in guidance of needle biopsy and ablation procedures of prostate cancer. Being able to determine this deformation is crucial for the registration of the interventionally acquired TRUS images to MR images acquired prior to the procedure [14].

The anatomical meshes were generated from a 320x320x15-voxel MR image with a $0.8 \times 0.8 \times 4 \mathrm{~mm}$ resolution with experimental, semi-automatic segmentation software and ANSYS $^{8}$. The deformable geometry of the simulation consists of two unconnected parts: the prostate consisting of 22,705 tetrahedra and 4425 nodes (purple in Fig. 12), and a block representing the surrounding tissue and the rectum consisting of 64,316 elements and 13,159 nodes (semi-transparent, blue in Fig. 12). The TRUS probe mesh was created in Meshlab and comprises 4886 triangular elements and 2445 nodes. Hu et al. [13] randomly sampled their material parameters from ranges given by $E \in[5,150] \mathrm{kPa}, \nu \in[0.3,0.4999]$ for the

\footnotetext{
8 http://www . ansys.com/
} 

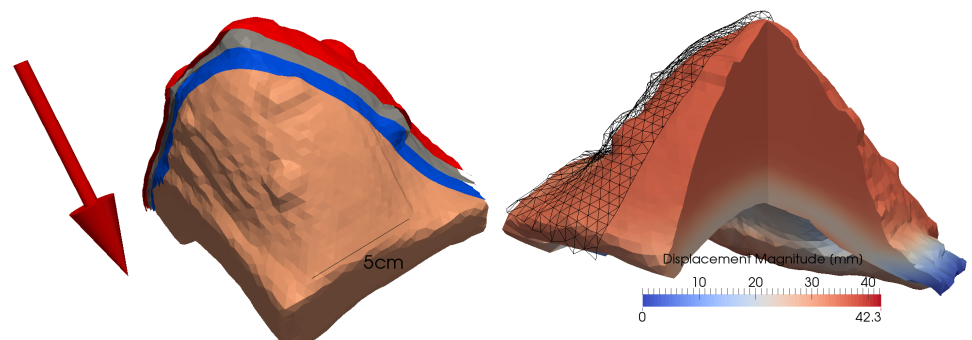

Fig. 13 Left: Initial configuration of the breast simulation with the skin contact assembly partially peeled away for better visibility, showing the outer contact surface (red), midplane (grey, not used in contact modelling), and the bottom part of the membrane contact assembly (blue), and the breast solid mesh (beige). The red arrow indicates the direction of gravity. Right: Superior-medial view of solid mesh and skin mid-surface final configurations with the skin mid-surface shown as wireframe mesh.

Young's modulus and Poisson ratio of the prostate components and $E \in[5,100] \mathrm{kPa}$ and $\nu \in[0.25,0.499]$ for the surrounding tissue, and used an inhomogeneous model for the prostate and the surrounding tissue. The parameter values chosen for this purely demonstrational simulation are identical for the prostate and the other tissue and set to $G=1.8 \mathrm{kPa}$, $K=6.9 \mathrm{kPa}$. The front and back face of the block are fixed in all spatial directions via displacement boundary conditions. The probe is translated by $(-0,-11.5,5) \mathrm{mm}$ from its initial to final position, in a linear motion. The simulation runs for a total of 1000 time steps, representing $1 \mathrm{~s}$ of pseudo time.

The second example is motivated by the registration of preoperatively acquired prone MR images used for planning of breast conserving cancer surgery to intra-operatively acquired supine MR images [10]. Sliding between the skin and underlying tissues has been observed but not properly quantified, having a method that allows for the modelling of this behaviour could therefore be used in future biomechanics-based registration algorithms for this type of application. In this example, the skin is modelled with a separate membrane mesh. The solid mesh comprises 37,613 tetrahedral elements and 10,614 nodes, and was generated from a $256 \times 512 \times 32$-voxel MR image with a $0.7 \times 0.7 \times 3.7 \mathrm{~mm}$ resolution with experimental segmentation software and TetGen ${ }^{9}$. The membrane mesh was generated by extraction of the surface of the solid mesh, offsetting by $3 \mathrm{~mm}$, and performing a manual segmentation; it has 4425 elements, 2283 nodes. The thickness of the shell elements is set to $5 \mathrm{~mm}$ leaving a $0.5 \mathrm{~mm}$ gap between the two meshes, that is quickly closed by the applied gravity forces. The chest-wall side of the solid mesh is prevented from moving vertically, and fully fixed along a narrow strip near the anatomically superior edge. The skin mesh is only held in the superior, lateral corner. The solid mesh is modelled as homogeneous transversely isotropic neo-Hookean [10] with $G=3.57 \mathrm{kPa}, K=16.67 \mathrm{kPa}, \eta=37.71 \mathrm{kPa}^{10}$ and the preferential direction coinciding with the anatomical anterior-posterior axis. The skin's material parameters are $E=25 \mathrm{kPa}, \nu=0.4$ for the membrane component, $E=5 \mathrm{kPa}, \nu=0.25$ for the bending stiffness. The simulation comprises 2500 time steps, representing 2.5 seconds of (pseudo) time.

The deformation in the first experiment (Fig. 12) is, as can be expected, quite localised with the TRUS probe penetrating into the block by about $1.1 \mathrm{~cm}$. In the process the prostate is

\footnotetext{
9 http://tetgen.berlios.de/

$10 \eta$ controls the stiffness in the preferred material direction, details can be found in the NiftySim user manual.
} 


\begin{tabular}{c||c|c} 
& Prostate & Breast \\
\hline BVH \& contact surface update & $0.3 \mathrm{~s}$ & $3.09 \mathrm{~s}$ \\
\hline Contact search & $45.55 \mathrm{~s}$ & $122.3 \mathrm{~s}$ \\
\hline Response Computation & $0.35 \mathrm{~s}$ & $0.15 \mathrm{~s}$ \\
\hline Other FEM operations & $40.63 \mathrm{~s}$ & $59.6 \mathrm{~s}$ \\
\hline \hline Total sim. computation time & $86.83 \mathrm{~s}$ & $185.15 \mathrm{~s}$
\end{tabular}

Table 5 Computation times for the breast and prostate image guidance examples broken down into the major stages of the contact-modelling pipeline

primarily rotated but also slightly bent with respect to its apex-base axis with the base being displaced by about $4 \mathrm{~mm}$. Two small dents made by the surrounding tissue displaced by the probe can also be seen on the prostate's posterior surface, where the peak displacement magnitude reaches $5.1 \mathrm{~mm}$. That the non-rigid deformation of the prostate isn't larger can probably be attributed to the mesh consisting of two parts that can slide relative to each other.

The deformation of the solid mesh in the breast example (Fig. 13) is primarily a compression in anterior-posterior direction combined with a shift of a sizeable portion of the mass in inferior and medial direction. The skin mesh is well held in place by the former despite there only being displacement boundary conditions on one corner of the mesh. Since the maximum displacement of the skin mid-surface is $2 \mathrm{~mm}$ greater than that of the solid mesh and by the extent by which the skin mesh is stretched over the solid mesh, a large amount of sliding between the two can be assumed. Further, due to the geometry of the problem, roughly half of both the solid mesh and skin contact surface can be assumed to be subject to contact constraints for most of the duration of the simulation, which is a larger fraction than in most simulations. Thus, it can be assumed that the contact modelling costs are higher in this simulation than in most simulations with comparable mesh resolutions.

However, the sum of the timings (Tab. 5) obtained for all contact modelling related operations is in both cases of the same magnitude as the time required for the basic FEM modelling which due to the low computational costs associated with the matrix-free approach of TLED is quite challenging in its own right.

\section{Conclusions}

We have presented methods suitable for detecting and handling of contacts arising in explicit FEM simulation of a range of scenarios: deformable geometry self-collisions, contacts between deformable solid and membrane meshes, and deformable geometry and a range of rigid geometry. The contact search portion of the presented pipeline is optimised for the typically small time-steps one has with explicit time integration in that it keeps the number of $\mathrm{BV}$ refittings low by identifying the parts of the BVH where containment of the geometry is ensured and self-collisions can be excluded. The success of this strategy can be seen in the consistently low numbers of BV refittings.

Further, in this paper, an improved formula for the computation, via Provot's recursive algorithm, of surface-normal bounding cones used for self-collision detection has been presented. We have demonstrated that the proposed formula leads to a marked reduction in the number of BVH subtrees that must be checked for self-collisions, compared to the formula originally proposed by Provot.

On the contact modelling side, we have presented a robust method that can deal with both geometric as well as temporal singularities via smoothing. Mathematically, the contact- 
force smoothing is, with respect to space, done by means of linearly interpolated surface normals, and with respect to time, by means of linearly increasing gap-rate proportional forces. In experiments, it has been shown that this approach improved simulation stability.

We have also shown that the entire proposed contact-modelling pipeline can be executed within a time frame that is of the same order of magnitude as the time required for standard TLED computations, in real-world image-guidance applications.

\section{Acknowledgement}

This work was partially funded by the PASSPORT Liver (EU FP7) grant, and in part by the Intelligent Imaging Programme Grant (EPSRC Reference: EP/H046410/1).

Sebastien Ourselin receives funding from the EPSRC (EP/H046410/1, EP/J020990/1, EP/K005278), the MRC (MR/J01107X/1), the EU-FP7 project VPH-DARE@IT (FP7-ICT-2011-9-601055), the NIHR Biomedical Research Unit (Dementia) at UCL and the National Institute for Health Research University College London Hospitals Biomedical Research Centre (NIHR BRC UCLH/UCL High Impact Initiative).

Conflict of interest S.F. Johnsen, Z.A. Taylor, L. Han, Y. Hu, M.J. Clarkson, D.J. Hawkes, and S. Ourselin declare that they have no conflict of interest.

\section{References}

1. J. Allard, S. Cotin, F. Faure, P. J. Bensoussan, F. Poyer, C. Duriez, H. Delingette, and L. Grisoni. SOFAan open source framework for medical simulation. Studies in health technology and informatics, 125:138, January 2007.

2. J. Allard, F. Faure, H. Courtecuisse, F. Falipou, C. Duriez, and P.G. Kry. Volume contact constraints at arbitrary resolution. ACM Transactions on Graphics, 29(4):1, July 2010.

3. M. Baumhauer, M. Feuerstein, H.P. Meinzer, and J. Rassweiler. Navigation in endoscopic soft tissue surgery: perspectives and limitations. Journal of endourology / Endourological Society, 22(4):751-66, April 2008.

4. D.M. Cash, M.I. Miga, S.C. Glasgow, B.M. Dawant, L.W. Clements, Z. Cao, R.L. Galloway, and W.C. Chapman. Concepts and preliminary data toward the realization of image-guided liver surgery. Journal of gastrointestinal surgery : official journal of the Society for Surgery of the Alimentary Tract, 11(7):84459, July 2007.

5. C. Duriez, F. Dubois, A. Kheddar, and C. Andriot. Realistic haptic rendering of interacting deformable objects in virtual environments. IEEE transactions on visualization and computer graphics, 12(1):36-47, 2006

6. F. G. Flores and E. Oñate. Improvements in the membrane behaviour of the three node rotation-free BST shell triangle using an assumed strain approach. Computer Methods in Applied Mechanics and Engineering, 194(6-8):907-932, February 2005.

7. S. Gottschalk, M.C. Lin, and D. Manocha. OBBTree: A hierarchical structure for rapid interference detection. In Proceedings of the 23rd annual conference on Computer graphics and interactive techniques, pages 171-180. ACM, 1996.

8. K. Gupta and A.P. Pobil. Practical motion planning in robotics: current approaches and future directions. Wiley, 1998

9. J.O. Hallquist. LS-DYNA Theory Manual. Technical report, Livermore, CA: Livermore Software Technology Corporation, 2006.

10. L. Han, J. Hipwell, Z.A. Taylor, C. Tanner, S. Ourselin, and D. Hawkes. Fast deformation simulation of breasts using GPU-based dynamic explicit finite element method. Digital Mammography, pages 728735,2010 .

11. B. Heidelberger, M. Teschner, and M. Gross. Detection of collisions and self-collisions using imagespace techniques. Journal of WSCG, 12(3):145-152, 2004.

12. M. Heinstein. Contact-impact modeling in explicit transient dynamics. Computer Methods in Applied Mechanics and Engineering, 187(3-4):621-640, July 2000.

13. Y. Hu, H. U. Ahmed, Z. Taylor, C. Allen, M. Emberton, D. Hawkes, and D. Barratt. MR to ultrasound registration for image-guided prostate interventions. Medical image analysis, 16(3):687-703, April 2012.

14. Y. Hu, T. J. Carter, H. U. Ahmed, M. Emberton, C. Allen, D. J. Hawkes, and D. C. Barratt. Modelling prostate motion for data fusion during image-guided interventions. IEEE transactions on medical imaging, 30(11):1887-900, November 2011. 
15. S.F. Johnsen, Z.A. Taylor, M. Clarkson, S. Thompson, M. Hu, K. Gurusamy, B. Davidson, D.J. Hawkes, and S. Ourselin. Explicit contact modeling for surgical computer guidance and simulation. In David R. Holmes III and Kenneth H. Wong, editors, Proc. SPIE 8316, Medical Imaging 2012: Image-Guided Procedures, Robotic Interventions, and Modeling, pages 831623-831623-9, February 2012.

16. S.F. Johnsen, Z.A. Taylor, Clarkson M.J., J. Hipwell, M. Modat, B. Eiben, L. Han, Y. Hu, T. Mertzanidou, D.J. Hawkes, and S. Ourselin. NiftySim: A GPU-based nonlinear finite element package for simulation of soft-tissue biomechanics. International Journal of Computer Assisted Radiology and Surgery, In press.

17. T.L. Kay and J.T. Kajiya. Ray tracing complex scenes. In ACM SIGGRAPH Computer Graphics, volume 20, pages 269-278. ACM, 1986.

18. T. Larsson and T. Akenine-Möller. Collision detection for continuously deforming bodies. Eurographics 2001, pages 325-333, 2001

19. T. Larsson and T. Akenine-Möller. A dynamic bounding volume hierarchy for generalized collision detection. Computers \& Graphics, 30(3):450-459, June 2006

20. B. Lee. Physically Based Modelling for Topology Modification and Deformation in Surgical Simulation. $\mathrm{PhD}$ thesis, University of Sydney, 2007.

21. M.C. Lin and S. Gottschalk. Collision detection between geometric models: A survey. In In Proc. of IMA Conference on Mathematics of Surfaces, pages 37-56, 1998.

22. J. Mezger, S. Kimmerle, and O. Etzmuß. Hierarchical techniques in collision detection for cloth animation. Journal of WSCG, 11(2):322-329, 2003.

23. K. Miller, G. Joldes, and D. Lance. Total Lagrangian explicit dynamics finite element algorithm for computing soft tissue deformation. in numerical methods in biomedical engineering, 23(2):121-134, 2007.

24. T. Möller and B. Trumbore. Fast, minimum storage ray/triangle intersection. In ACM SIGGRAPH 2005 Courses, page 7. ACM, 2005.

25. M.A. Otaduy, R. Tamstorf, D. Steinemann, and M. Gross. Implicit Contact Handling for Deformable Objects. Computer Graphics Forum, 28(2):559-568, April 2009.

26. X. Provot. Collision and self-collision handling in cloth model dedicated to design garments. In Graphics interface, volume 97, pages 177-189. Citeseer, 1997.

27. M. Puso. A mortar segment-to-segment frictional contact method for large deformations. Computer Methods in Applied Mechanics and Engineering, 193(45-47):4891-4913, November 2004.

28. J.C. Simo. A finite strain beam formulation. The three-dimensional dynamic problem. Computer methods in applied mechanics and engineering, 49:55-70, 1985.

29. G Székely, C Brechbühler, R Hutter, A Rhomberg, N Ironmonger, and P Schmid. Modelling of soft tissue deformation for laparoscopic surgery simulation. Medical Image Computing and Computer-Assisted Interventation - MICCAI '98, 1496(1):550-561, March 1998.

30. L.M. Taylor and D.P. Flanagan. PRONTO 3D: A three-dimensional transient solid dynamics program. Technical Report SAND87-1912, Sandia National Laboratories, Albuquerque, NM, March 1989.

31. Z.A. Taylor, M. Cheng, and S. Ourselin. High-speed nonlinear finite element analysis for surgical simulation using graphics processing units. IEEE transactions on medical imaging, 27(5):650-663, May 2008.

32. Z.A. Taylor, O. Comas, M. Cheng, J. Passenger, D.J. Hawkes, D. Atkinson, and S. Ourselin. On modelling of anisotropic viscoelasticity for soft tissue simulation: numerical solution and GPU execution. Medical image analysis, 13(2):234-44, April 2009.

33. P. Volino and N. M. Thalmann. Efficient self-collision detection on smoothly discretized surface animations using geometrical shape regularity. Computer Graphics Forum, 13(3):155-166, August 1994.

34. P. Wriggers. Computational contact mechanics. J. Wiley \& Sons, 2002.

35. P. Wriggers and L. Krstulovič-Opara. On Smooth Finite Element Discretizations for Frictional Contact Problems. ZAMM - Journal of Applied Mathematics and Mechanics / Zeitschrift für Angewandte Mathematik und Mechanik, 80(S1):77-80, March 2000.

36. Bin Yang and Tod A. Laursen. A contact searching algorithm including bounding volume trees applied to finite sliding mortar formulations. Computational Mechanics, 41(2):189-205, September 2006. 